\title{
EDITORIAL
}

\section{Decreased muscle PPAR concentrations: a mechanism underlying skeletal muscle abnormalities} in COPD?

\author{
S.A. Sathyapala*, P. Kemp ${ }^{\#}$ and M.I. Polkey*
}

$\mathrm{P}$ eripheral muscle weakness is a common and serious complication of chronic obstructive pulmonary disease (COPD), which precedes loss of fat-free mass and is an independent predictor of mortality in patients with Global Initiative for COPD grade III or IV disease [1]. Current treatment options for muscle weakness are limited to exercise and supplemental feeding for cachectic patients. However, this approach is not effective in all individuals and the development of drugs that could be used as adjunctive therapies in the treatment of muscle weakness is hindered by a lack of knowledge of the molecular mechanisms responsible for the muscle dysfunction.

Two types of muscle abnormality are observed in the quadriceps muscle of patients with moderate and severe COPD. Atrophy occurs, particularly of the anaerobic type-IIx fibres, and is manifest as a loss of muscle strength. In addition, loss of muscle oxidative capacity arises from the depletion of aerobic type-I fibres and a reduction in mitochondria and oxidative enzymes within both type-I and -IIa fibres, resulting in decreased muscle endurance. At present, it is unclear whether these two processes are linked, for example whether atrophy of type-IIx fibres drives a switch of type-I to type-II fibres, or whether these processes occur independently. Animal studies have identified an array of pathways that could be involved in the development of muscle atrophy or loss of oxidative capacity. Some of these may be more relevant in animal models than in human disease. For example, some pathways that have been studied may be more involved in muscle development rather than a response to external stimuli, let alone the complex array of stimuli which may be present in COPD. In addition, there may be redundancy in the system so that multiple pathways have to be disrupted before skeletal muscle dysfunction arises. To date, few candidate molecules have been studied in humans and there has been little focus on mediators that could potentially link the processes of muscle

*Muscle Laboratory, Royal Brompton Hospital, and ${ }^{*}$ National Heart and Lung Institute, Imperial College London, London, UK.

SUPPORT STATEMENT: S.A. Sathyapala is the recipient of a Wellcome Trust (London, UK) Clinica Research Training Fellowship jointly supervised by M.I. Polkey and A.M.W.J. Schols, investigating the role of PPAR- $\boldsymbol{\delta}$ in skeletal muscle fibre type shift in COPD.

STATEMENT OF INTEREST: None declared.

CORRESPONDENCE: M.I. Polkey, Muscle Laboratory, Royal Brompton Hospital, Sydney Street, London, SW3 6NP, UK. Fax: 44 2083518939. E-mail: m.polkey@rbh.nthames.nhs.uk atrophy and loss of oxidative capacity, given that they frequently co-occur in disease.

In the present issue of the European Respiratory Journal, REMELS et al. [2] present a study of the expression of peroxisome proliferator-activated receptor (PPAR)- $\alpha$ and PPAR- $\delta$ in the quadriceps muscle of patients with moderate-to-very severe COPD compared with controls with a similar smoking history. REMELS et al. [2] also investigated mitochondrial transcription factor A (TFAM), a transcription factor required for mitochondrial gene expression, and PPAR- $\gamma$ coactivator (PGC- $1 \alpha$ ), which is also an activator of other transcription factors, such as nuclear respiratory factor (NRF)1 and myocyte enhancer factor (MEF)2c. Although the cohort was small (14 patients and nine controls), PPAR- $\delta$ protein, TFAM protein and PGC- $1 \alpha$ mRNA concentrations were found to be significantly lower in patients than in controls. The study by REMELs et al. [2] also found that concentrations of TFAM mRNA and protein and PPAR $-\alpha$ mRNA were significantly lower in the cachectic COPD patients than both the noncachetic patients and controls. PPAR $-\alpha$ and PPAR $-\delta$ mRNA concentrations correlated with levels of citrate synthase, which is an oxidative enzyme. Muscle PPAR- $\alpha$ mRNA concentrations inversely correlated with circulating levels of tumour necrosis factor (TNF)- $\alpha$ and soluble TNF- $\alpha$ receptors 55 and 75 .

These are interesting findings and we believe this is the first paper exploring these signalling pathways, which are highly plausible for the aetiology of fibre-type shift and muscle wasting in COPD. Naturally there are some limitations; the sample size was small and a larger cohort might have brought out significant decreases in all elements of the pathway. This type of cross-sectional approach works most efficiently if the investigated pathways are uniformly active throughout the patients' illness; in this case, the patients were clinically stable but it could be that atrophic signalling, for example, is particularly severe during certain clinical events, such as exacerbation. We also note that the control group had a slightly subnormal mean carbon monoxide diffusion capacity of $84 \%$ predicted and, like the patients, a slightly low mean arterial partial pressure of oxygen, raising the possibility that some controls had a modest degree of emphysema. However, the fact that patients and controls had similar arterial partial pressures of oxygen yet significant differences in PPAR- $\alpha$ and PPAR- $\delta$, PGC- $1 \alpha$ and TFAM expression suggests that hypoxaemia may not have a significant effect on expression of these molecules in vivo. 
The results are highly plausible in the context of previous studies. Disruption of PPAR- $\alpha$ and PPAR- $\delta$ activity occurs in in vitro conditions of hypoxia [3] and systemic inflammation [4], which are common complications of COPD. The pattern and magnitude of the fibre-type shift in COPD is the opposite of that seen in the transgenic "marathon mouse" model where activated PPAR- $\delta$ expression is increased [5]. Transgenic mice with increased expression of PGC- $1 \alpha$ in the skeletal muscle develop a greater proportion of type-I fibres in lower limb muscles than wild-type mice [6] and appear to be protected against muscle wasting mediated via the FoxO3/atrogin1 and muscle-specific RING finger protein 1 pathways [7].

In humans, reduced PGC- $1 \alpha$, PPAR- $\alpha$ and PPAR- $\delta$ activity has been demonstrated in other disease states with similar skeletal muscle abnormalities to COPD. For example, KRÄMER et al. [8] demonstrated that quadriceps PPAR- $\alpha$ and PPAR- $\delta$ mRNA concentrations are reduced in patients with spinal cord injury who had an anaerobic muscle phenotype compared with healthy controls and particularly elite endurance cyclists. In each of these three groups, PPAR- $\alpha$ mRNA concentration positively correlated with the proportion of type-I fibres, and PPAR- $\alpha$ and PPAR- $\delta$ concentrations were inversely proportional to the percentage of type-IIx fibres [8]. Other studies have demonstrated that muscle PGC- $1 \alpha$ concentrations are reduced in congestive cardiac failure [9] and diabetes mellitus [10]. Such studies suggest that the PGC-1 $\alpha /$ PPAR axis is disrupted in conditions characterised by a reduced muscle oxidative capacity, regardless of the primary disease.

If it is not a sample-size effect, the observation that PPAR- $\delta$ protein but not mRNA concentrations were decreased in patients with COPD suggests that there is post-translational modification of the PPAR molecule. As protein catabolism may be accelerated in COPD through the action of ubiquitin proteosome and calpain proteases, PPAR- $\delta$ may be specifically targeted for destruction in the muscle of patients. It would have been useful to have data on activity, not just the quantity, of the PPARs in patients versus controls, for example by chromatin immunoprecipitation to measure the quantity of PPAR bound to DNA, and by measuring products of PPARresponsive genes, such as carnitine palmitoyl transferase 1 and 2. Data on NRF1 expression would have completed the mapping of the PGC-1 $\alpha /$ NRF1/TFAM pathway [11].

PPAR- $\alpha$ mRNA muscle concentrations were lower in the cachetic group of COPD patients who had higher levels of circulating TNF- $\alpha$ and soluble TNF- $\alpha$ receptors than the noncachetic patients and controls. There is growing evidence that all three PPAR subtypes have an anti-inflammatory action and aged mouse models suggest that PPAR- $\alpha$ has bidirectional antagonism with the nuclear factor- $\kappa \mathrm{B}$ signalling pathway [12]. In addition, studies with PPAR- $\alpha$ null mice indicate that PPAR- $\alpha$ mediates the adaptive response to fasting by stimulating hepatic fatty acid uptake and oxidation and inhibiting enzymes in the urea cycle to limit protein breakdown [13]. Hence, we speculate that COPD patients with the highest levels of systemic inflammation, who are prone to muscle atrophy through a number of pathways, develop globally suppressed PPAR expression and, therefore, fail to conserve muscle in situations of negative energy balance and so develop cachexia. However, the role of inflammation in the expression of PPAR genes in skeletal muscle remains to be defined. A possible model of the role of PPARs in the development of skeletal muscle atrophy and loss of oxidative capacity in COPD is outlined in figure 1.

One has to be careful about drawing any conclusions about causation from observational studies. As PPAR- $\alpha$ and PPAR- $\delta$ are preferentially expressed in type-I fibres, it is entirely possible that disruption of other signalling pathways in the skeletal muscle of patients with COPD results in loss of muscle oxidative phenotype, and only as a consequence of this is PPAR expression reduced. However, if this was the case, it is still possible that PPAR upregulation/activation would reverse the fibre shift. Indeed, the ultimate test of the relevance of the current findings is a trial of highly selective PPAR- $\alpha$ and PPAR- $\delta$ agonists in patients with muscle dysfunction in COPD. Such agonists are currently in phase-I trials as treatment for dyslipidaemias.

In conclusion, REMELS et al. [2] report disruption of the peroxisome proliferator-activated receptor- $\gamma$ coactivator/peroxisome proliferator-activated receptor- $\alpha$ and peroxisome proliferator-activated receptor- $\delta$ and peroxisome proliferatoractivated receptor- $\gamma$ coactivator/mitochondrial transcription factor A axes, which are associated with skeletal muscle abnormalities in patients with chronic obstructive pulmonary disease. This is a novel and interesting finding that warrants further investigation in larger samples, with more detailed exploration of the signalling cascades involved and prospective intervention studies to determine the clinical triggers. Work such as this, directed towards elucidating mechanisms underpinning skeletal muscle dysfunction in chronic obstructive pulmonary disease as pharmacological targets, holds the key to improving exercise tolerance, health status and possibly

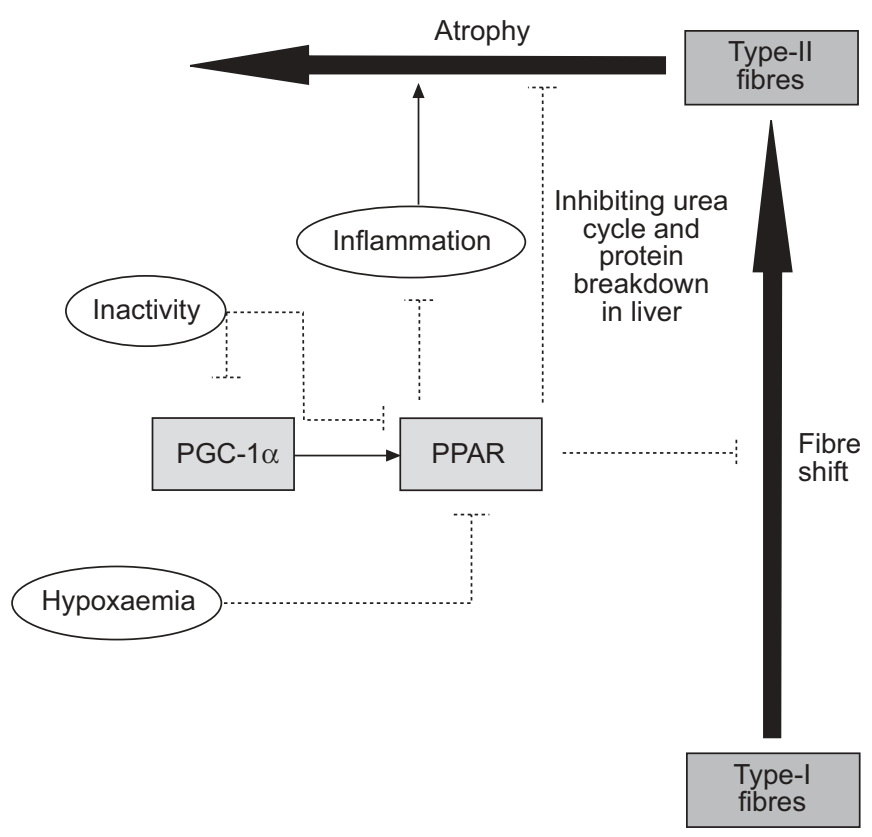

FIGURE 1. Proposed model of involvement of peroxisome proliferatoractivated receptors (PPAR) in the development of skeletal muscle abnormalities in chronic obstructive pulmonary disease. PGC- $1 \alpha$ : PPAR- $\gamma$ coactivator. ............ inhibitory effect. Arrows indicate activation. 
mortality for the millions of chronic obstructive pulmonary disease patients worldwide. It is also likely to have implications for sufferers of other chronic disorders with similar skeletal muscle manifestations.

\section{REFERENCES}

1 Swallow EB, Reyes D, Hopkinson NS, et al. Quadriceps strength predicts mortality in patients with moderate to severe chronic obstructive pulmonary disease. Thorax 2007; 62: 115-120.

2 Remels AH, Schrauwen P, Broekhuizen R, et al. Peroxisome proliferator-activated receptor expression is reduced in skeletal muscle in COPD. Eur Respir J 2007; 30: 245-252.

3 Narravula S, Colgan SP. Hypoxia-inducible factor 1mediated inhibition of peroxisome proliferator-activated receptor- $\alpha$ expression during hypoxia. J Immunol 2001; 166: 7543-7548.

4 Beier K, Volkl A, Fahimi HD. TNF- $\alpha$ downregulates the peroxisome proliferator activated receptor $\alpha$ and the mRNAs encoding peroxisomal proteins in rat liver. FEBS Lett 1997; 412: 385-387.

5 Wang YX, Zhang CL, Yu RT, et al. Regulation of muscle fiber type and running endurance by PPAR $\delta$. PLOS Biol 2004; 2: e294.
6 Lin J, Wu H, Tarr PT, et al. Transcriptional co-activator PGC- $1 \alpha$ drives the formation of slow-twitch muscle fibres. Nature 2002; 418: 797-801.

7 Sandri M, Lin J, Handschin C, et al. PGC- $1 \alpha$ protects skeletal muscle from atrophy by suppressing FoxO3 action and atrophy-specific gene expression. Proc Natl Acad Sci USA 2006; 103: 16260-16265.

8 Krämer DK, Ahlsén M, Norrbom J, et al. Human skeletal muscle fibre type variations correlate with PPAR $\alpha$, PPAR $\delta$ and PGC-1 $\alpha$ mRNA. Acta Physiol (Oxf) 2006; 188: 207-216.

9 Garnier A, Fortin D, Deloménie C, Momken I, Veksler V, Ventura-Clapier R. Depressed mitochondrial transcription factors and oxidative capacity in rat failing cardiac and skeletal muscles. J Physiol 2003; 551: 491-501.

10 Roden M. Muscle triglycerides and mitochondrial function: possible mechanisms for the development of Type 2 diabetes. Int J Obes (Lond) 2005; 29: Suppl. 2, S111-S115.

11 Scarpulla RC. Nuclear control of respiratory chain expression in mammalian cells. J Bioenerg Biomemb 1997; 29: 109-119.

12 Poynter ME, Daynes RA. Peroxisome proliferator-activated receptor- $\alpha$ activation modulates nuclear factor- $\kappa \mathrm{B}$ signalling, and reduces inflammatory cytokine production in ageing. J Biol Chem 1998; 273: 32833-32841.

13 Kersten S, Seydoux J, Peters JM, Gonzalez FJ, Desvergne B, Wahli W. Peroxisome proliferator-activated receptor- $\alpha$ mediates the adaptive response to fasting. J Clin Invest 1999; 103: 1489-1498. 